\title{
La Tour de la Bondue à Autun. Un exemple de résidence urbaine fortifiée en Bourgogne, $\mathrm{XIII}^{\mathrm{e}}$ - $\mathrm{XV}^{\mathrm{e}}$ siècle
}

Mémoire de master 2, sous la direction de Dany Sandron, université de Paris-Sorbonne (Paris IV), soutenue en septembre 2012

Julien Barthel

\section{OpenEdition}

1 Journals

Édition électronique

URL : https://journals.openedition.org/cem/12966

DOI : $10.4000 /$ cem. 12966

ISSN : 1954-3093

Éditeur

Centre d'études médiévales Saint-Germain d'Auxerre

Référence électronique

Julien Barthel, « La Tour de la Bondue à Autun. Un exemple de résidence urbaine fortifiée en

Bourgogne, $x \mathrm{III}^{\mathrm{e}}-\mathrm{xv}$ e siècle », Bulletin du centre d'études médiévales d'Auxerre | BUCEMA [En ligne], 17.1 |

2013, mis en ligne le 03 juin 2013, consulté le 22 septembre 2022. URL : http://

journals.openedition.org/cem/12966; DOI : https://doi.org/10.4000/cem.12966

Ce document a été généré automatiquement le 22 septembre 2022.

\section{(c) (i) (2) (2)}

Creative Commons - Attribution - Pas d'Utilisation Commerciale - Partage dans les Mêmes Conditions 4.0 International - CC BY-NC-SA 4.0

https://creativecommons.org/licenses/by-nc-sa/4.0/ 


\section{La Tour de la Bondue à Autun. Un exemple de résidence urbaine fortifiée en Bourgogne, $\mathrm{XIII}^{\mathrm{e}}$ - $\mathrm{XV}^{\mathrm{e}}$ siècle}

Mémoire de master 2, sous la direction de Dany Sandron, université de Paris-Sorbonne (Paris IV), soutenue en septembre 2012

\section{Julien Barthel}

La Tour de la Bondue est une de ces constructions mal connues, même pour bon nombre d'habitants d'Autun. Cette situation résulte de l'urbanisation importante qui, au $\mathrm{xIX}^{\mathrm{e}}$ siècle, a progressivement enclavé les constructions médiévales, les rendant moins visibles dans le paysage urbain, tout en freinant les études architecturales. C'est avec Charles Boëll, dans les années 1920, que la première analyse de la tour est réalisée ${ }^{1}$. Il s'agit avant tout d'une recherche axée sur l'histoire du bâtiment et plus encore sur ses propriétaires, mais l'auteur avance déjà des datations. Selon lui, la tour aurait été construite au $\mathrm{XIII}^{\mathrm{e}}$ siècle. En prenant

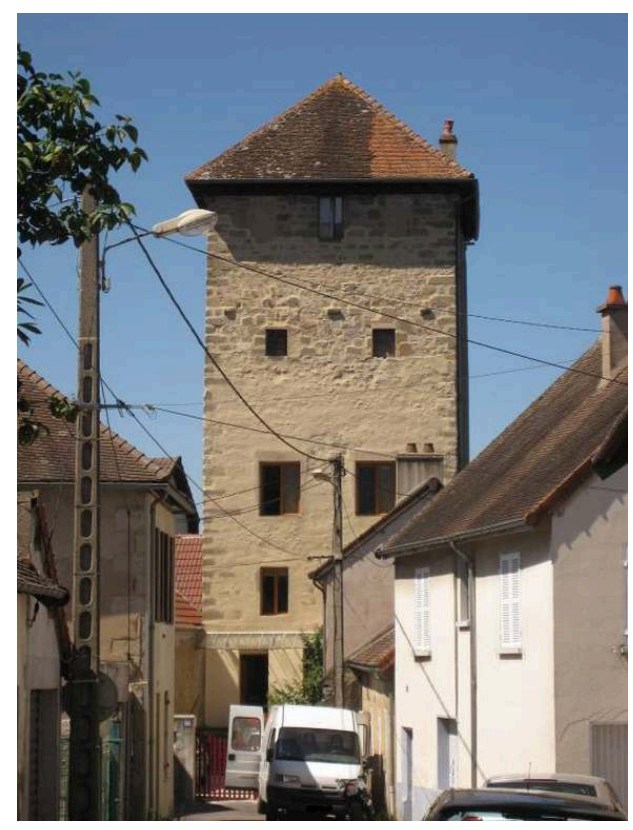
appui sur un plan dressé par un architecte au XVIII ${ }^{\mathrm{e}}$ siècle (fig. 1), il affirme également que ce bâtiment faisait partie d'un complexe plus important. De nos jours, seule la tour subsiste. 
Fig. 1 - «Plan des cazernes de la ville d'Autun », plan de Joubert, 1785, C 43 (cl. Sylvie BalconBerry).

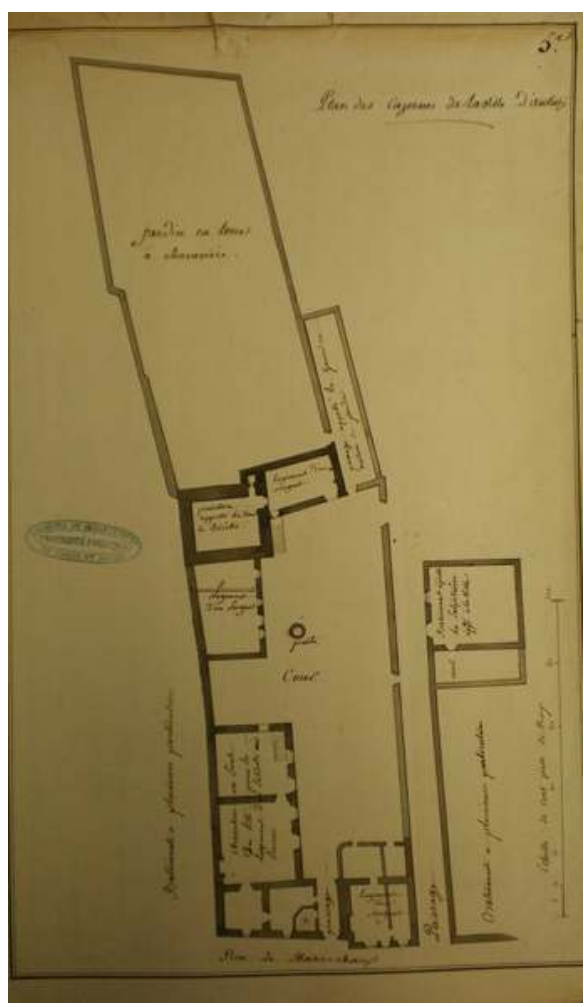

2 De cette étude, nous retenons que les plus anciennes mentions conservées de la tour sont issues d'un acte notarié de vente du 30 novembre 1606, donc bien postérieur à la période médiévale ${ }^{2}$. Cet acte mentionne toutefois le rachat du bâtiment par un certain Jean Le Maire aux héritiers de Guillaume Boisserant, le 4 avril 1475. Bien qu'il ne cite pas ses sources, Charles Boëll précise que la famille Garin ou Guérin possède la tour aux environs de 1431 et qu'elle est vendue à ce Guillaume Boisserant à la même époque. Ce dernier est alors conseiller du duc de Bourgogne et ancien gouverneur de la viérie d'Autun.

3 Par la suite, ce sont les Le Maire, seigneurs de la Bondue, qui, comme nous l'avons vu, achètent le complexe et lui donne son nom. Il le garde jusqu'au 19 février 1600 lorsque les possessions sont achetées par Claude Perreault, greffier à la chancellerie au siège de Chalon-sur-Saône. Celui-ci les vend peu de temps après, le 14 mai 1605, à Pierre Jeannin, président du parlement de Bourgogne, conseiller du roi de France et baron de Chagny.

4 Un an plus tard, les bâtiments sont vendus à son cousin André Jeannin. Par la suite, la maison de la Bondue est vendue à d'autres propriétaires qui la louent. Le 5 juillet 1727, elle est finalement acquise par la ville d'Autun pour être transformée en casernes, destinées à loger des troupes de passage et des garnisons. La maison est divisée le 16 mars 1777. La ville ne conserve que la tour utilisée comme chambre de sûreté ; elle la vend au mois de mars 1840.

5 Cette tour de plan rectangulaire, d'environ $8,50 \mathrm{~m}$ par $7 \mathrm{~m}$, est haute d'environ $17,50 \mathrm{~m}$ hors charpente. Sur les deux tiers de sa hauteur, elle est construite en moellons. Ses angles sont renforcés par un chaînage de pierres de taille. Le tiers restant est, quant à lui, constitué de pierres en moyen et grand appareil (fig. 2). 
Fig. 2 - La Tour de la Bondue, face sud (cl. Julien Barthel).

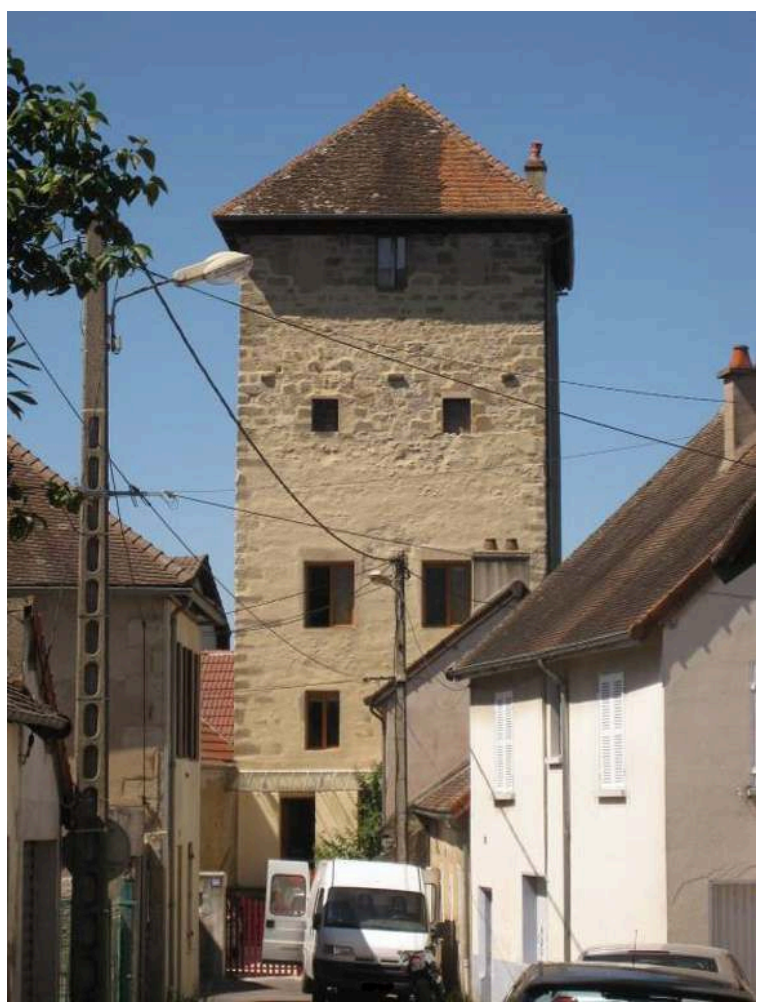

6 Elle s'élève sur six niveaux dont une cave ou glacière semi-enterrée, les cinq niveaux supérieurs sont desservis par un escalier interne en bois situé dans l'angle nord-ouest. La tour se trouve dans le quartier de Marchaux, au centre de la ville (fig. 3), dévolu principalement au commerce, comme l'attestait la présence de grandes halles en bois aujourd'hui détruites. 
Fig. 3 - Autun, voies, remparts et monuments à la fin du Moyen Âge (le point rouge indique la position de la tour de la Bondue).

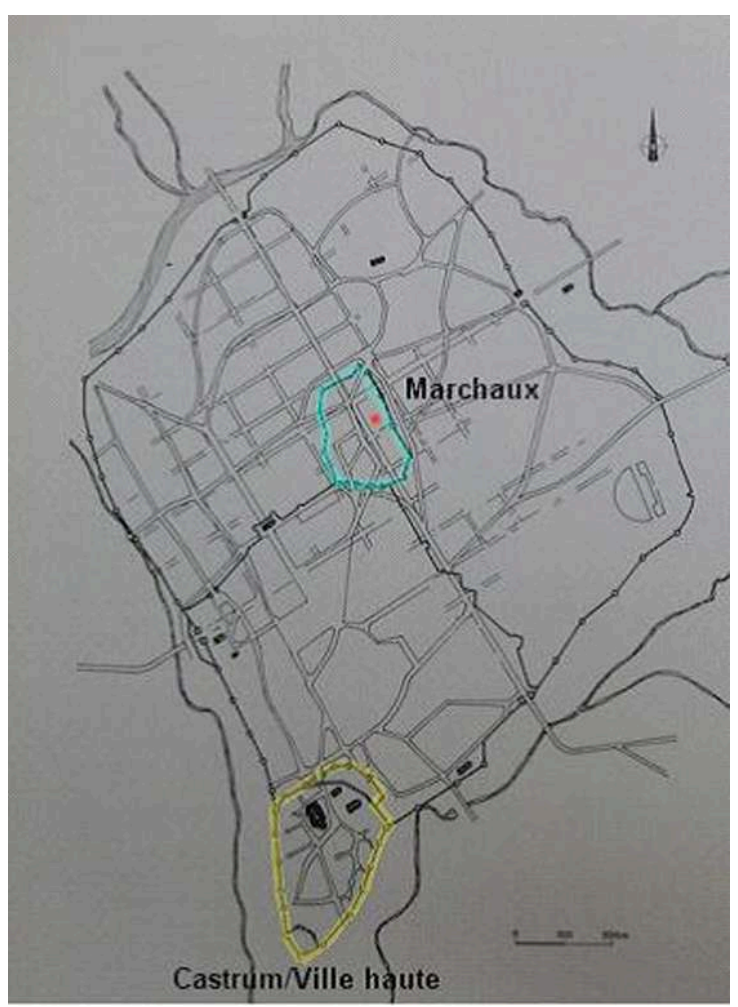

7 Des travaux de rénovation entrepris par les propriétaires actuels ont rendu accessible une partie des maçonneries internes ${ }^{3}$. Les observations réalisées au cours du suivi de ces travaux, associées à des relevés pierrent à pierre ${ }^{4}$, ont permis d'identifier un certain nombre d'éléments et surtout d'en proposer une séquence chronologique (fig. 4,5 et 6$)^{5}$. Ainsi, il a été possible de déterminer au moins trois phases de construction certaines et une probable.

Fig. 4 - Tour de la Bondue, parement extérieur du mur sud (dessin J. Barthel). 


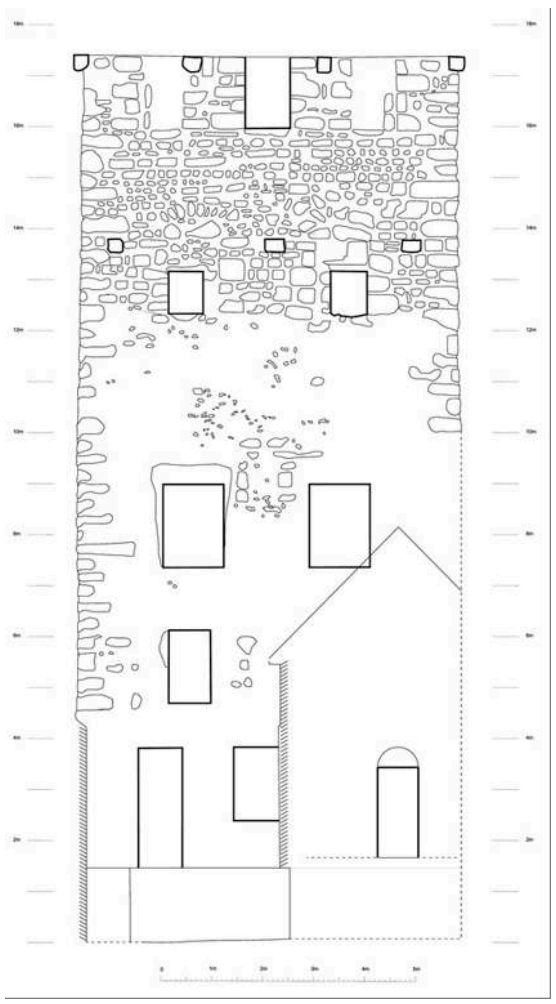

Fig. 5 - Tour de la Bondue, parement extérieur du mur ouest (dessin J. Barthel).

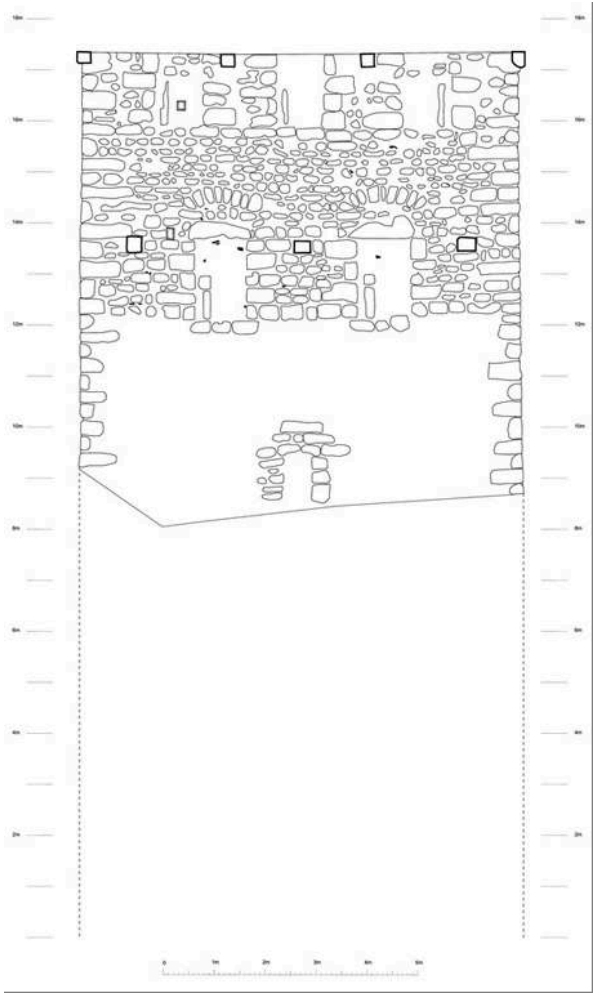


Fig. 6 - Tour de la Bondue, parement extérieur du mur est (cl. J. Barthel).

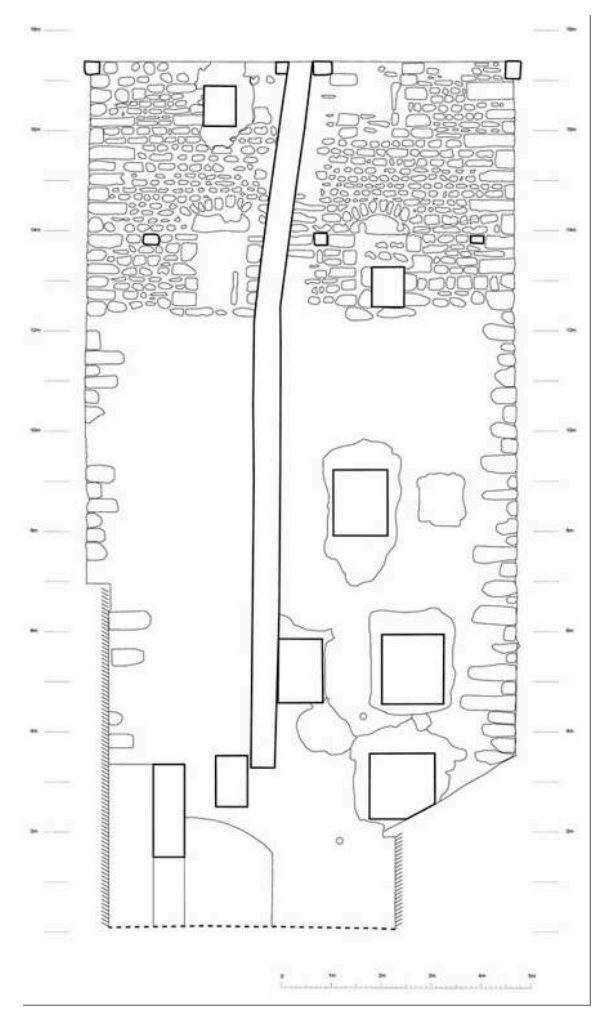

8 La première phase est difficilement identifiable, car un certain nombre de modifications réalisées au $\mathrm{xx}^{\mathrm{e}}$ siècle ont brouillé, voire empêché, la lecture des maçonneries. Elle comprend la base de la tour jusqu'à environ $2,50 \mathrm{~m}$ du sol, jusqu'à l'assise située au-dessus de la clé d'un arc bouché dans le mur est. Il est impossible de la dater, mais elle est clairement antérieure à la deuxième phase.

Cette deuxième phase est bien plus visible. Elle commence donc à partir de 2,50 m jusqu'aux corbeaux visibles sur les quatre faces, à environ $14 \mathrm{~m}$ du sol, c'est-à-dire du $2^{\mathrm{e}}$ niveau à la première partie du $5^{\mathrm{e}}$ niveau. Cette phase peut être datée du XIII ${ }^{\mathrm{e}}$ siècle, car ce bâtiment vient s'insérer dans un ensemble de constructions typologiquement et chronologiquement proches et surtout caractéristiques de la Bourgogne. Hervé Mouillebouche parle d'ailleurs de "tours bourguignonnes" pour caractériser ces constructions ${ }^{6}$. Ces édifices se distinguent par leur plan carré ou rectangulaire, et une hauteur de 2 à 5 étages. Leur origine probable serait ces maisons urbaines à étages surélevés, comme à Tournus ou à Vézelay.

En l'absence de documents écrits, il est impossible de proposer une date plus précise pour cette phase. Au XIII ${ }^{\mathrm{e}}$ siècle, la tour s'élevait ainsi sur trois niveaux en plus de la cave. Le premier étage, englobant le deuxième et le troisième niveau actuel, devait avoir une fonction équivalente à celle d'une aula castrale. Cette grande salle comportait, par ailleurs, la seule cheminée de la tour. Le deuxième niveau était percé de quatre petites fenêtres et de latrines, ce qui conduit à l'assimiler à une chambre à coucher. Enfin, le dernier étage servait à l'observation et à la défense. Il comprenait deux ouvertures carrées situées juste sous la charpente, appelées " créneaux » par les auteurs médiévaux. Ce dernier niveau est d'ailleurs la partie la plus caractéristique de ce genre de constructions. Hervé Mouillebouche indique que ces baies sont pratiquées afin de faire tenir les toits de lave, utilisée en Bourgogne, sur des éléments solides 7 . 
11 La troisième phase consiste en un exhaussement de la tour d'un étage. Le cinquième niveau est tout d'abord surélevé. Par souci de solidité du bâti, les ouvertures rectangulaires sont surmontées d'arcs surbaissés destinés à supporter le poids du dernier étage. Celui-ci équivaut au précédent, si ce n'est que ses murs sont percés de trois « créneaux ». La fonction de ces derniers niveaux reste inchangée : il s'agit d'un étage double de défense. Cette surélévation a probablement eu lieu entre 1356 et 1453. La première date correspond à l'ordonnance de la mise en défense du duché de Bourgogne ${ }^{8}$; la seconde date signe la fin de la guerre de Cent Ans. Ce nouvel étage avait en effet pour but d'améliorer le système défensif du bâtiment, tout en fournissant une meilleure surveillance des abords du quartier de Marchaux. On peut même conjecturer que cette reprise se situe plus précisément entre 1358, lorsque les habitants commencent à réparer les remparts ${ }^{9}$, et 1364 , quand le château de la Vesvre, proche d'Autun, tombe aux mains des routiers.

La quatrième phase n'appartient pas à la période médiévale, mais est essentielle à la compréhension du bâtiment. La tour n'est plus un lieu d'habitation. Les fenêtres sont réduites puis, pour un grand nombre d'entre elles, bouchées, tout comme la cheminée. Ces modifications laissent penser que l'édifice était utilisé comme lieu de stockage. Par ailleurs, la grande salle est divisée en deux dans sa hauteur avec l'adjonction d'un nouveau plancher, qui, aujourd'hui, sépare toujours les deuxième et troisième niveaux. C'est également lors de ces travaux que les planchers sont refaits et que l'escalier médiéval est remplacé par l'actuel. Les travaux ont commencé au cours du XvII siècle, comme en témoignent les planchers à la française, typiques de cette période. Il est probable qu'en 1840 lorsque la ville vend la tour, les fenêtres sont bouchées.

Si la fonction de chaque niveau semble acquise, il subsiste la question de la place de la tour dans la ville. Sur le plan architectural, elle est trop modeste pour jouer un rôle défensif majeur, ses murs dépassant à peine un mètre d'épaisseur. Par ailleurs, aucune archère n'a été aménagée, ce qui est particulièrement révélateur du peu d'importance du bâtiment dans le système de défense de la ville basse. Enfin, les étages défensifs ne sont guère efficaces, car les " créneaux » empêchent tout tir plongeant. En définitive, il semble que cette tour était avant tout un poste d'observation. À plus de 15 mètres de haut, la vue était particulièrement dégagée et suffisamment bonne pour pouvoir avertir les habitants des dangers potentiels. Lors de la guerre de Cent Ans, cette tour correspondait peut-être au bâtiment le plus haut de Marchaux. En effet, il devait être plus économique de surélever une tour de cette importance que d'en construire une nouvelle. Se pose également le problème du propriétaire. Il s'agissait peut-être d'un noble de Marchaux jouissant d'un pouvoir particulier, qui devait jouer un rôle officiel dans la défense du quartier, tel le bailli dans la ville haute.

Reçu : 5 mars 2013 - Accepté : 24 avril 2013

\section{NOTES}

1. C. BoËLl, Autun, notices et documents, Archives de la Société éduenne, M. 53, p. 530-537. 
2. Acte de vente de la seigneurie de la Bondue du 30 novembre 1606, Archives de la Société éduenne, série I, Autun, rue de la Bondue.

3. Je remercie chaleureusement la famille Lagneau qui m'a permis de suivre les travaux et qui m'a autorisé à faire les relevés. Certains relevés avaient été entrepris en 2004 par Christophe Besnier, alors étudiant en DEA à l'université Paris-Sorbonne.

4. Je tiens à remercier également le service archéologique d'Autun, notamment Yannick Labaune et Angélique Tisserand, pour leur assistance technique lors du relevé des murs extérieurs. Merci aussi à Sylvie Balcon-Berry, sans qui cette étude n'aurait pu aboutir, et à André Strasberg, conservateur au musée Rolin et secrétaire perpétuel de la Société éduenne.

5. J. BARTHEL, La Tour de la Bondue à Autun, étude archéologique d'une résidence urbaine fortifiée en Bourgogne, XII ${ }^{e}$-XVe siècle, mémoire de master 2, sous la direction de D. SANDRON, université de Paris IV Sorbonne, 2012.

6. H. MOUILLEBOUCHE, Les maisons fortes en Bourgogne du nord, du XIII au XVI esiècle, Dijon, 2002, p. 152.

7. H. MOUILLEBOUCHE, Les maisons fortes..., ibid., p. 215.

8. H. MOUILleBOUCHE, Les maisons fortes..., ibid., p. 329.

9. A. DE CHARMASSE, « Précis historique », in Autun et ses monuments, Autun, 1889, p. 167.

INDEX

Index géographique: France/Autun

Mots-clés : tour, résidence urbaine

\section{AUTEUR}

\section{JULIEN BARTHEL}

Université de Bourgogne, UMR Artehis 